\title{
On the role of otoliths and semicircular canals in spatial orientation: Dynamics of the visually perceived eye level during gondola centrifugation
}

\author{
ARNE TRIBUKAIT and OLA EIKEN \\ Karolinska Institute and Swedish Defense Research Agency, Stockholm, Sweden
}

\begin{abstract}
The visually perceived eye level (VPEL) was measured during gondola centrifugation. Subjects $(N=$ 11) were seated upright, facing motion in a swing-out gondola. The head was adjusted so that Reid's baseline was tilted $10^{\circ}$ anterior end up. The subjects were requested to adjust the position of a small luminous dot so that it was perceived as gravitationally at eye level. In the 1-g environment, the VPEL was a few degrees below the true gravitational eye level $\left(M=-1.75^{\circ}, S D=1.90^{\circ}\right)$. After rapid acceleration of the centrifuge to $2 \mathrm{G}$ (vectorial sum of the earth gravity force and the centrifugal force), there was an exponentially increasing depression of the VPEL. The initial value was $-6.4^{\circ} \pm 5.2^{\circ}$. During 10 min at $2 \mathrm{G}$, the VPEL approached an asymptotic value of $-24.8^{\circ} \pm 5.4^{\circ}$. The time constant showed a large interindividual variability, ranging from 59 to $1,000 \mathrm{sec}(M=261 \mathrm{sec}$, median $=147 \mathrm{sec})$. The findings are discussed, taking into consideration otolith-semicircular-canal interaction, as well as memory functions of the vestibular system.
\end{abstract}

Virtually all perceiving and thinking has a spatial component. Orientation in space denotes the ability of an individual to perceive the position and motion of his or her own body, as well as of objects in the surroundings, with respect to a frame of reference. The ever-present gravity vector provides a direction to our image of the surroundings. The vestibular receptors of the inner ear play an important role in spatial orientation by sensing the head's position with respect to gravity, as well as angular and linear head movements. This idiothetic information makes the perception of the orientation or movement of visual objects relatively independent of the individual's own motor activities. For the study of spatial orientation, it is often useful to consider a simple quantifiable component, such as the perceived vertical or perceived eye level. How such variables are influenced by stimuli to the sense of balance may then shed light on the relationship between different functions of the vestibular system and our ability to orient in space.

The otolith organs (the utricle and the saccule) sense linear accelerations of the head, as well as the gravity force. The effective stimulus is the gravitoinertial shear force component acting in the plane of the macula (Fernandez, Goldberg, \& Abend, 1972; Loe, Tomko, \& Werner, 1973). This causes a displacement of the otoconial membrane, relative to the sensory epithelium, which will bend the sensory hairs of the receptor cells. The utricular

The authors thank Michael Crisp and Bertil Lindborg for technical assistance and Erik Dahlman for performing calculations. Correspondence concerning this article should be addressed to A. Tribukait, Defense Research Agency, Berzelius v 13, Karolinska Institutet, SE 17177 Stockholm, Sweden (e-mail: arne.tribukait@foi.se). macula is oriented in approximately the same plane as the lateral (horizontal) semicircular canal (Lindeman, 1969), tilted rostrally upward $30^{\circ}$ with respect to Reid's baseline (a line from the external auditory meatus to the inferior margin of the orbit; Corvera, Hallpike, \& Schuster, 1958). Thus, when the head is held in its natural upright position, the utricle is tilted rostrally upward by approximately $40^{\circ}$. The saccular macula is vertically oriented in the parasagittal plane (Lindeman, 1969). It is elongated, and the length axis is approximately parallel with the plane of the utricular macula (Corvera et al., 1958; Naganuma, Tokumasu, Okamoto, Hashimoto, \& Yamashina, 2001).

For any head position with respect to gravity, there will be a specific pattern of shearing forces acting in the planes of the utricular and saccular maculae. For instance, when the head is tilted backward, there is an increase in the posteriorly directed shear force component acting in the plane of the utricular macula, which changes the activity patterns in neural afferents from different regions of the macula. Such vestibular information contributes to the ability to perceive a stationary visual object as keeping its position regardless of changes in head position with respect to gravity.

Many studies on the influence of the vestibular organs on spatial orientation have employed a small luminous target at a straight-ahead position in front of the test subject. The subject is required to adjust the target, in what is otherwise complete darkness, so that it appears to be at eye level. The angular deviation from the true eye level is recorded. This quantitative measure of orientation in pitch has been termed the visually perceived eye level (VPEL; DiZio, Li, Lackner, \& Matin, 1997; Matin \& Fox, 1989). For normal subjects, seated upright in the 1 -g environ- 
ment, the VPEL is usually a few degrees below the true gravitational eye level (M. M. Cohen, 1973; DiZio et al., 1997; Hoppeler, 1913).

When the resultant gravitoinertial force is increased, a subject who is seated upright will experience an illusion of being tilted backward (Schöne, 1964). Concomitantly, a stationary visual target, at eye level in front of the subject, is perceived as being moved upward (Beckh, 1956; Clark, Graybiel, \& MacCorquodale, 1948; M. M. Cohen, 1973). These illusions are due to the pitched-up orientation of the utricular macula in the skull. The increase in gravitoinertial force results in a posteriorly directed shear of the otoconial membrane equivalent to that caused by a posterior head tilt in the 1-g environment (Schöne, 1964). A quantitative estimate of these effects may be obtained by measurements of the VPEL. When asked to set the luminous target at the perceived eye level, the subject sets it below the true eye level; the deviation is considered to be, to a large extent, dependent on the shear force component acting in the plane of the utricular macula (Correia, Hixson, \& Niven, 1968; Schöne, 1964).

However, in many situations, the semicircular canals influence the perception of changes in head orientation. If a change in head position with respect to gravity is made rapidly, the subjective estimate of tilt will be larger than if the angular speed is below the threshold of the canals (Stockwell \& Guedry, 1970). On the other hand, there are certain conditions in which the different parts of the vestibular organ do not provide unanimous information about changes in head orientation with respect to gravity. An example is the pattern of movements experienced during acceleration in a swing-out gondola centrifuge. Due to the swing-out of the gondola during acceleration, the direction of angular velocity stimulation of the semicircular canals will gradually change from the yaw (horizontal) plane to near the pitch (sagittal) plane (at $2 \mathrm{G}$, the gondola inclination is $60^{\circ}$ ). Hence, if the subject is sitting upright and facing forward, he or she will experience an increasing pitch-backward rotation. It is not clear whether this angular velocity component of the stimulus contributes to the illusion of backward tilt, which is induced by the increased gravitoinertial force.

Most studies on the relationship between vestibular stimulation and changes in VPEL have considered the tonic effects mediated via the otolith organs. Contamination of the results by temporary semicircular canal activity has generally been avoided by using very low angular accelerations or by starting the data collection after a certain period of constant velocity.

The aim of the present investigation was to study the influence on VPEL of the complex semicircular canal stimulation that occurs during acceleration in a gondola centrifuge. Special attention was paid to interaction between the semicircular canals and the otolith organs, as well as on vestibular memory functions. These problems were addressed by asking test subjects to make repetitive indications of the VPEL during a time period of at least $10 \mathrm{~min}$ after a rapid acceleration of the centrifuge.

\section{METHOD}

\section{Test Subjects}

Thirteen healthy subjects, 5 females and 8 males, 20-37 years of age, were recruited for the study. A few days before testing, they had taken part in another centrifuge experiment and had experienced the same stimulus conditions as those in the present study. They did not have any history of vertigo, hearing loss, or neurological disturbances and were free from medications. The subjects participated with their informed consent and were free to withdraw at any time during the experiment. The test procedure was in accordance with the Declaration of Helsinki and was approved by the Local Human Ethics Committee.

\section{Centrifugation}

The experiments were performed in the human-use centrifuge at the Karolinska Institute, Stockholm. The radius of the centrifuge is $7.25 \mathrm{~m}$. A swing-out gondola is fixed on a pivot whose axis is tangential, so that the gondola is deflected outward in the direction of the resultant force vector (resultant of earth gravity force and the centrifugal force). The subjects were fixed in a cockpit seat by means of safety belts. The head position was fixed by use of a head holder. It was adjusted so that a line from the external auditory meatus to the inferior margin of the orbit was tilted upward (nose up) $10^{\circ}$ with respect to the gravitational horizontal. The interocular line was gravitationally horizontal. No bite bar was used, due to the necessity of being able to communicate with the subjects during the runs and also in order to minimize discomfort; in case of anxiety or motion sickness, a bite bar may increase the discomfort.

The rotation of the centrifuge is counterclockwise (as seen from above), with the subject facing forward (see Figure 1). During centrifuging, the vertical axis of the gondola and, hence, the long axis $(z$-axis) of the subject are always aligned with the resultant force vector of the earth gravity force and the centrifugal force. The pivot does not permit any change in pitch position of the gondola. With an angular acceleration of $15 \% \mathrm{sec}^{2}$, the settled gravitoinertial force level of $2 \mathrm{G}$ was attained in $6 \mathrm{sec}$.

All recording equipment was located in the experimenter's room, separated from the centrifuge well. The subject was observed in infrared light by means of a video camera, and he or she always had the possibility of communicating with the experimenter by means of a two-way intercom system. The subject's heart rate and rhythm were monitored continuously by means of electrocardiography.

\section{Measurements of the VPEL}

In front of the subject (at a distance of $0.8 \mathrm{~m}$ from the subject's eyes), there was a screen on which a small red laser dot might be projected. The laser pointer was mounted to the right of the subject's head on a horizontal axle, which coincided with a line passing through the center of the eyes. The axle was connected to a low voltage DC motor and a digital angle encoder (Heidenhain ERN 1080), making it possible to adjust the direction of the pointer in the pitch plane. The screen formed a circular segment in the pitch plane, so that the distance between the laser dot and the subject's eyes was not influenced by the deviation of the dot from the true eye level position. Each time the dot appeared on the screen, the subject had to adjust it, by means of two pushbuttons on a remote control, so that it appeared to be at eye level. When pleased with a setting, the subject pressed a third button, which extinguished the dot. The deviation relative to the gravitoinertial eye level was recorded with an accuracy of $0.1^{\circ}$. Before the laser pointer was turned on again, the experimenter changed its direction (randomly) $5^{\circ}-20^{\circ}$, alternately up and down. Except for the laser dot, the gondola was completely darkened.

The subject was instructed to imagine that he or she was sitting on a beach, observing the horizon of the sea. The luminous dot should be adjusted so that it was at the same height as the horizon. If he or 


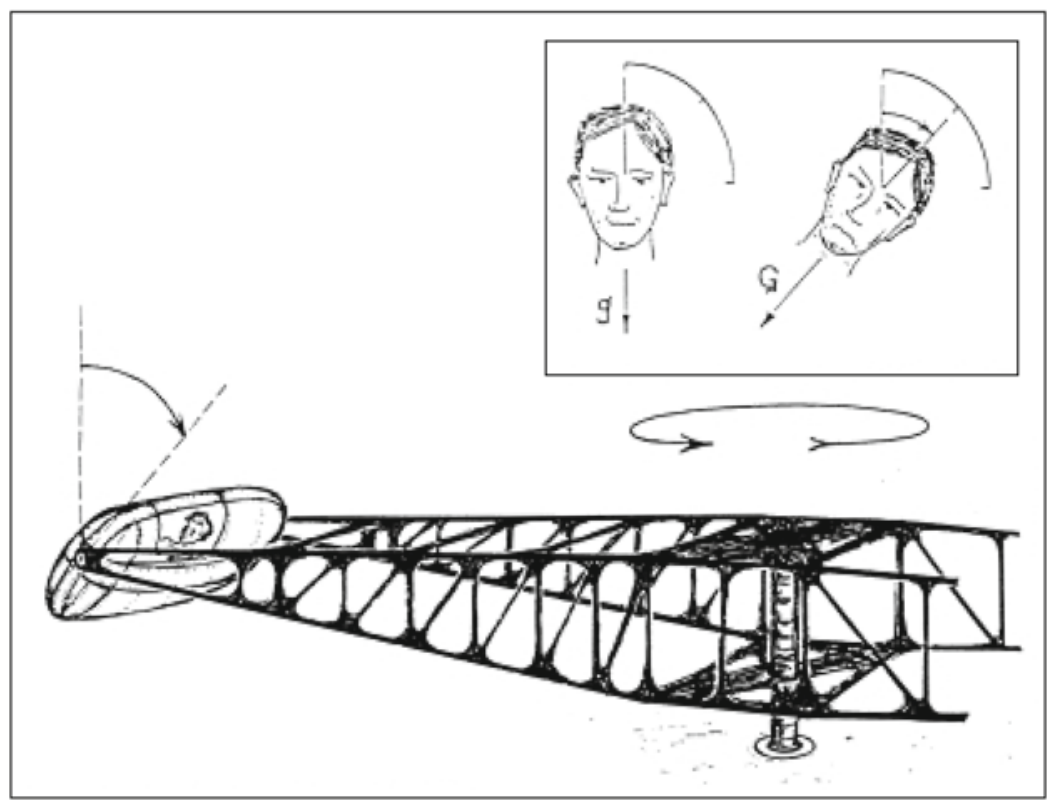

Figure 1. The centrifuge. Rotation of the centrifuge is counterclockwise, as seen from above, with the subject facing forward. During acceleration, the cabin is rolled so that the resultant gravitoinertial force vector (resultant of the earth gravity and the centrifugal force) remains aligned with the subject's head-to-seat $(z)$ axis.

she had an impression of head and body tilt, he or she should simply indicate, by adjusting the luminous dot, the horizon of the plane in relation to which he or she felt tilted, rather than the perceived direction of his or her head-sagittal (antero-posterior) axis.

Prior to centrifugation, when the subject had been fastened and the gondola darkened, an initial series of four settings of the laser dot was performed. During centrifugation, data collection was started within $5 \mathrm{sec}$ after the end of acceleration of the centrifuge. The data collection continued for at least $10 \mathrm{~min}$.

\section{RESULTS}

One of the subjects did not understand the instructions correctly. For another subject, the data were very different from those of the other subjects, and the possibility of a technical error could not be excluded. Thus, the data presented below were obtained from 11 subjects (denoted 1-11).

Individual data for the VPEL at $1.0 \mathrm{~g}$ (prior to centrifugation) are shown in Figure 2. The deviation of the VPEL from the true gravitational horizontal is given in degrees. If VPEL was above the true eye level, it has been denoted by a positive sign; if below, it has been denoted by a negative sign. All except 1 of the subjects had a VPEL that was slightly below the true eye level. The group mean was $-1.75^{\circ} \pm 1.90^{\circ}(S D)$, which is significantly different from zero (one-sample $t$ test: $p=.01 ; n=11$ ). For most individuals, the variability, expressed as $1 S D$ of four settings of the luminous dot, was smaller (the group mean of $1 S D$ of four settings was $1.0^{\circ} ; n=11$ ) than the interindividual variability ( $1 S D$ of the 11 means was $\left.1.90^{\circ} ; n=11\right)$. This might suggest that the interindividual variability is not due to uncertainty of the test subjects but reflects individual differences in a stable system for sensing the direction of gravity in the pitch plane.

After acceleration of the centrifuge, at $2 \mathrm{G}$, all the subjects experienced a sensation of being tilted backward. One of them reported the feeling that her head was tilted backward, while the body was still upright. For all the subjects, there was a downward deviation of the VPEL. This deviation gradually increased and seemed to stabilize, by the end of data collection, about an asymptotic value.

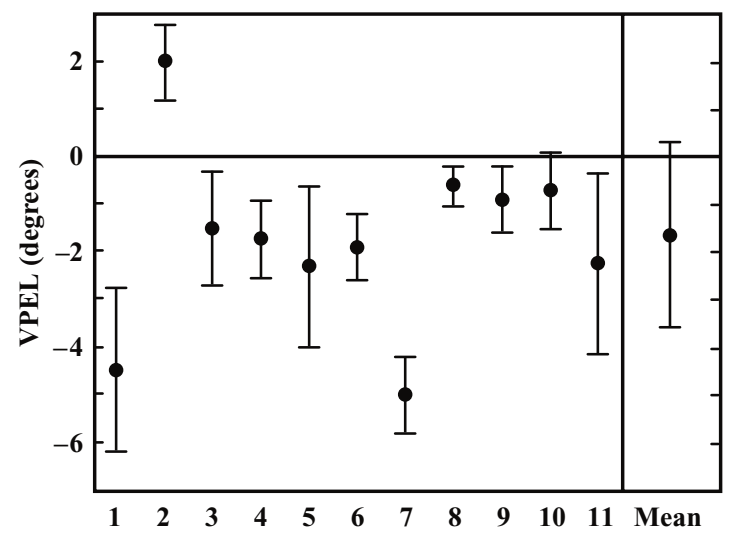

Figure 2. The visually perceived eye level (VPEL) in the 1-g environment. The mean value and one standard deviation of four settings are shown for each individual. In most subjects, the VPEL was slightly below the true gravitational eye level. The variability within subjects was smaller than the interindividual variability $(1 S D ; n=11)$. 
No. 1

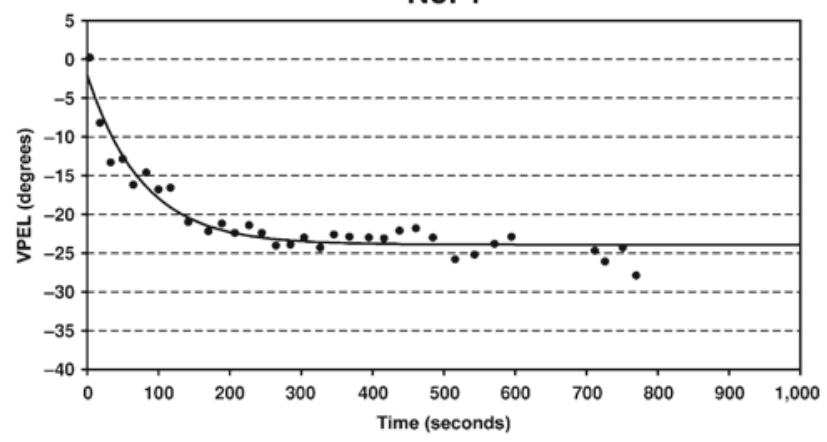

No. 3

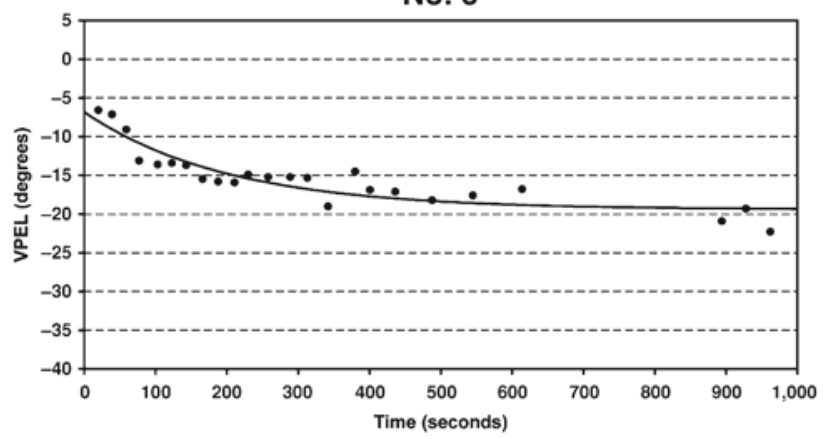

No. 2

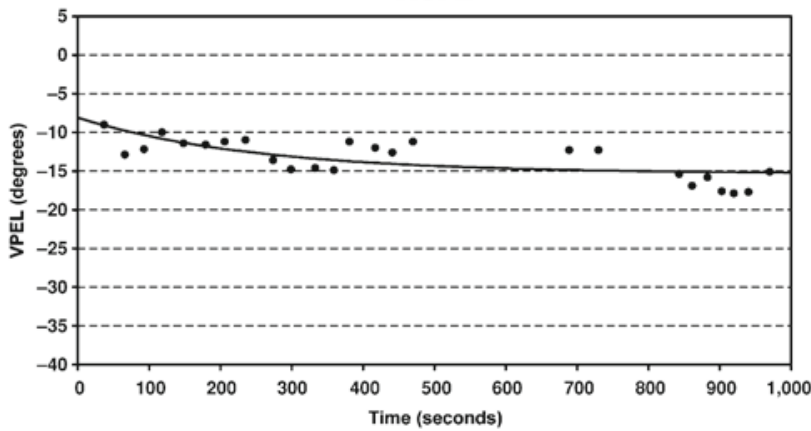

No. 4

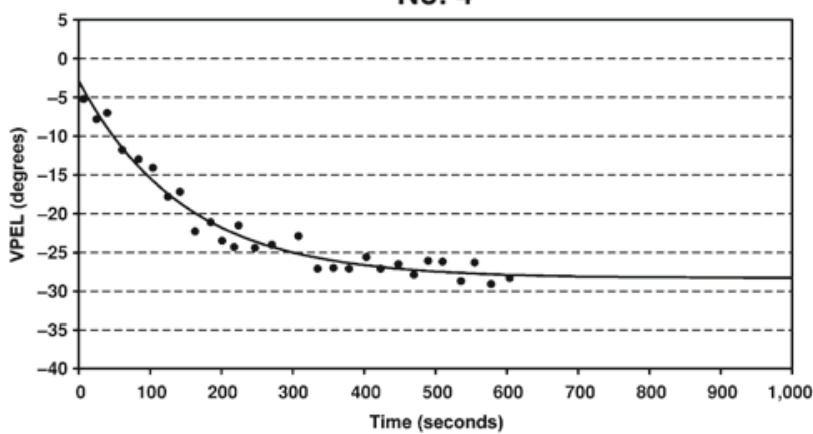

Figure 3. Individual diagrams for the changes with time of the visually perceived eye level (VPEL). Each point represents one setting of the luminous dot. The negative value corresponds to a VPEL that is below the true gravitoinertial eye level. The lines represent best-fitting exponential functions.

Figure 3 shows how the VPEL changes with time at $2 \mathrm{G}$ in the 11 subjects. For each individual, an exponential function, VPEL $=A e^{-b t}+C$, has been adapted to the data (least square fit). $A+C$ equals the value for VPEL at the time $t=0$ (end of angular acceleration of the centrifuge). The time constant, $T$, for exponential decay is obtained as $T=1 / b . C$ is the asymptote. Group mean values for each 30 -sec interval, as well as curve fitting to group data, are presented in Figure 4.

Individual data for the constants characterizing the changes in VPEL are shown in Table 1. At the time $t=0$, the mean VPEL was $-6.4^{\circ}$, and the median was $-6.6^{\circ}$ ( $\left.1 S D=5.2^{\circ} ; n=11\right)$. The mean asymptotic value was $-24.8^{\circ}$, and the median was $-24.8^{\circ}\left(1 S D=5.4^{\circ} ; n=\right.$ 11 . For the time constant, $T$, the interindividual variability was large. A few subjects had a time constant of $300-1,000 \mathrm{sec}$. The group mean was $261 \mathrm{sec}$, the median was $147 \mathrm{sec}$, and $1 S D$ was $280 \mathrm{sec}$.

There was no relationship between the VPEL in a 1-g environment and the initial value at $2 \mathrm{G}$ (linear regression: $r=.13, p=.71 ; n=11)$. However, despite the limited number of subjects, there tended to be a correlation between the VPEL at $1 \mathrm{~g}$ and the asymptotic value at $2 \mathrm{G}$ (linear regression: $r=.59, p=.055 ; n=11$; see Figure 5). This might suggest that both the VPEL at $1 \mathrm{~g}$ and the asymptote at $2 \mathrm{G}$ are measures mainly of graviceptive function, whereas the initial value for VPEL at $2 \mathrm{G}$ must be, to a great extent, influenced by the semicircular canals.

\section{DISCUSSION}

\section{Graviceptive Stimulation and the Asymptotic Value for the VPEL}

The asymptotic value for the VPEL is most likely a pure measure of graviceptive function; that is, it is independent of semicircular canal function. A mean value of $-24.8^{\circ}$ for the upright head position at $2 \mathrm{G}$ is consistent with the data reported by Schöne (1964), as well as by Correia et al. (1968). In these earlier reports, an increase in the posteriorly directed shear force component acting in the plane of the utricle was suggested to be a major cause of the depression of the VPEL in hypergravity. Hence, when the head was pitched nose down by $30^{\circ}$, bringing the utricular macula into a gravitationally horizontal position, the influence on the VPEL of changes in the G force was very minor (Correia et al., 1968; Schöne, 1964).

However, this does not mean that the VPEL is dependent solely on utricular input. There could, in principle, be other factors contributing to, or counteracting, the hypergravityinduced depression of the VPEL. M. M. Cohen (1973) proposed neck proprioceptors to be one such factor. According to the theories developed by Mittelstaedt, the VPEL is determined by utricular and saccular information, as well as by an idiotropic vector - that is, a tendency to localize the perceptual zenith so that it coincides with the head and body length axis. Whereas the utricular signal, resulting from the posteriorly directed macular shearing, causes a depression of the VPEL, information from the saccule, as well as the 


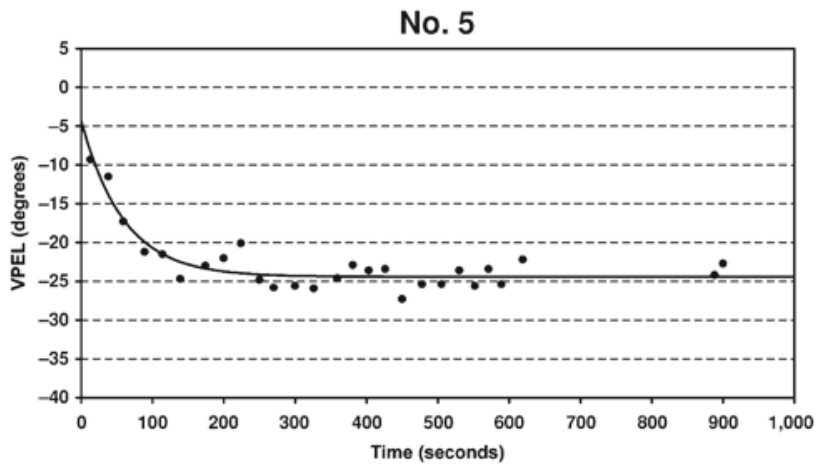

No. 7

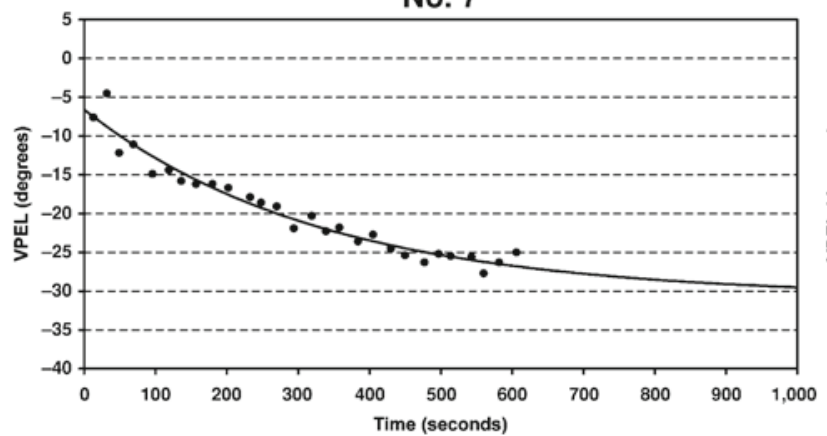

No. 6

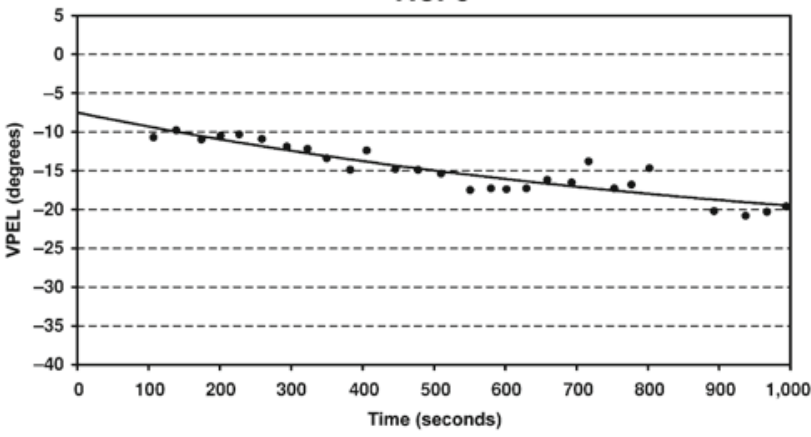

No. 8

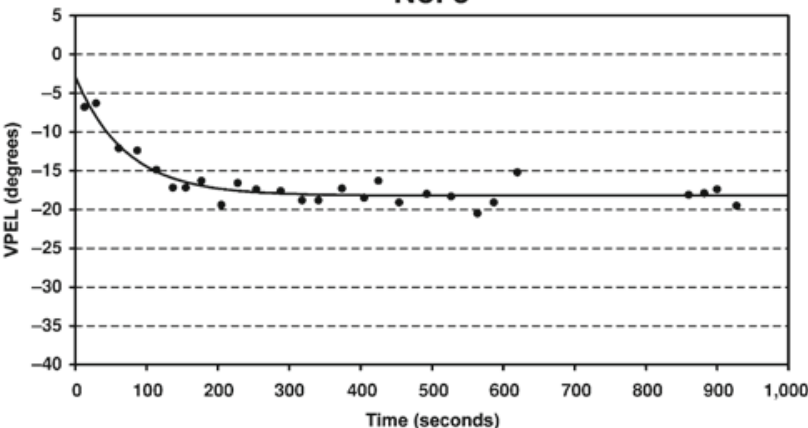

Figure 3. (Continued).

idiotropic vector, tends to minimize this depression (for further details, see Mittelstaedt, 1983, 1988, 1989, 1995).

The saccular macula is oriented in the parasagittal plane so that its length axis is parallel with the plane of the utricular macula - that is, tilted rostrally upward by $30^{\circ}$ with respect to Reid's baseline (Corvera et al., 1958, Naganuma et al., 2001). It is divided by the S-shaped striola into a superior and an inferior area. In both areas, the
No. 9

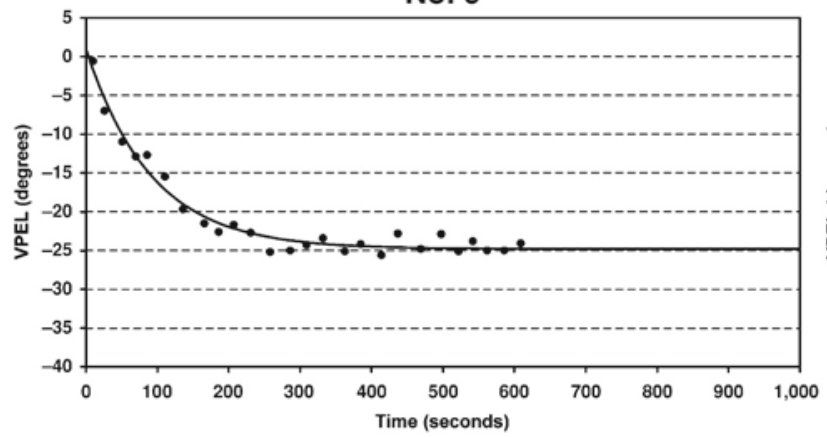

No. 11

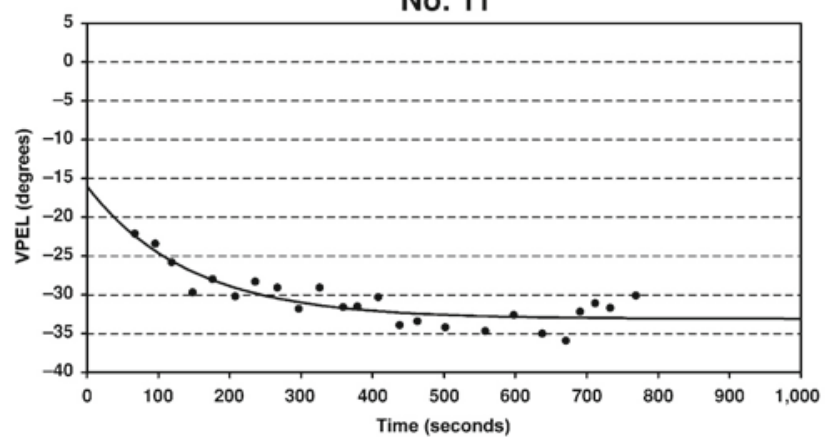

No. 10

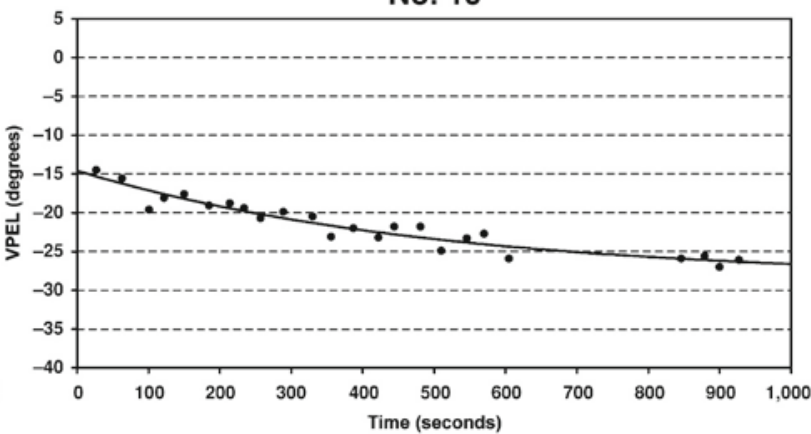

Figure 3. (Continued). 


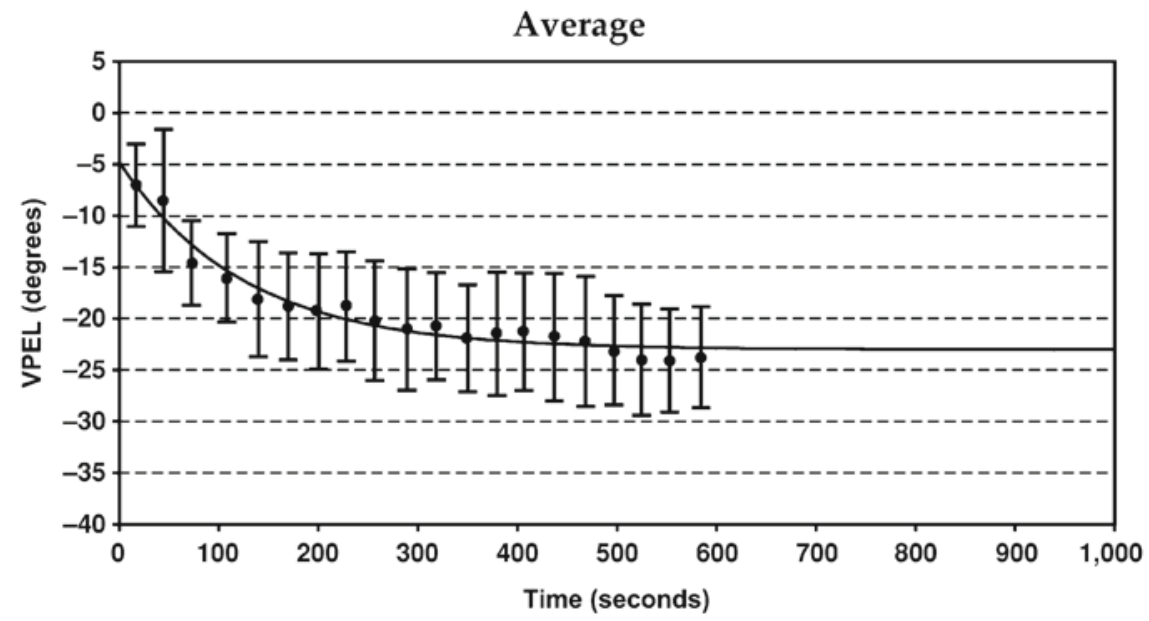

Figure 4. Changes with time of the visually perceived eye level (VPEL) for the group of subjects. For every 30-sec interval, the group mean $(N=11)$ has been calculated. The data points represent mean values for both the VPEL and the point of time. In cases in which an individual had made two settings during a time interval, the mean of these two settings was first calculated. Error bars represent $1 S D$. As regards time, $1 S D$ (not shown) was between 4 and 9 sec for every interval. The line represents the best-fitting exponential function.

hair cells are excitatorily stimulated by otoconial displacement away from the striola. Due to the curved shape of the striola, there are hair cells with functional polarization vectors in practically all directions in the pitch plane. For a given head position in the pitch plane, there will be a specific pattern of stimulation of the hair cells in different regions of the macula, some being maximally excited, others being maximally inhibited. The pattern will be similar irrespectively of the magnitude of the $\mathrm{G}$ vector. Thus, although the utricle will overestimate changes in head position when the $\mathrm{G}$ force is increased, there is the possibility that the saccule gives a correct estimate of head position in pitch even at $2 \mathrm{G}$.

As regards the neck proprioceptors, these may, theoretically, cooperate with abdominal graviceptors (Mit-

Table 1

The VPEL at $1 \mathrm{~g}$ and the Constants Characterizing the Change in VPEL With Time at $2 \mathrm{G}$ for Each Individual

\begin{tabular}{|c|c|c|c|c|}
\hline Subject & $\begin{array}{l}\text { VPEL } \\
\text { at } 1 \mathrm{~g}\end{array}$ & $\begin{array}{l}\text { Initial } \\
\text { Value } \\
\text { at } 2 \mathrm{G} \\
(A+C)\end{array}$ & $\begin{array}{c}\text { Asymptote } \\
\text { at } 2 \mathrm{G} \\
\text { (C) }\end{array}$ & $\begin{array}{c}\text { Time } \\
\text { Constant } \\
\quad(\mathrm{sec}) \\
(T=1 / b)\end{array}$ \\
\hline 1 & $-4.6^{\circ}$ & $-2.0^{\circ}$ & $-23.9^{\circ}$ & 77 \\
\hline 2 & $+1.9^{\circ}$ & $-8.1^{\circ}$ & $-15.3^{\circ}$ & 250 \\
\hline 3 & $-1.5^{\circ}$ & $-6.8^{\circ}$ & $-19.4^{\circ}$ & 200 \\
\hline 4 & $-1.7^{\circ}$ & $-2.9^{\circ}$ & $-28.3^{\circ}$ & 147 \\
\hline 5 & $-2.3^{\circ}$ & $-4.3^{\circ}$ & $-24.4^{\circ}$ & 59 \\
\hline 6 & $-1.8^{\circ}$ & $-7.5^{\circ}$ & $-26.5^{\circ}$ & 1,000 \\
\hline 7 & $-5.0^{\circ}$ & $-6.6^{\circ}$ & $-30.7^{\circ}$ & 333 \\
\hline 8 & $-0.6^{\circ}$ & $-2.9^{\circ}$ & $-18.2^{\circ}$ & 71 \\
\hline 9 & $-0.9^{\circ}$ & $+1.0^{\circ}$ & $-24.8^{\circ}$ & 91 \\
\hline 10 & $-0.6^{\circ}$ & $-14.6^{\circ}$ & $-28.5^{\circ}$ & 500 \\
\hline 11 & $-2.2^{\circ}$ & $-16.0^{\circ}$ & $-33.1^{\circ}$ & 143 \\
\hline Mean & $-1.75^{\circ}$ & $-6.4^{\circ}$ & $-24.8^{\circ}$ & 261 \\
\hline Median & $-1.70^{\circ}$ & $-6.6^{\circ}$ & $-24.8^{\circ}$ & 147 \\
\hline $1 S D$ & $1.90^{\circ}$ & $5.2^{\circ}$ & $5.4^{\circ}$ & 280 \\
\hline
\end{tabular}

telstaedt, 1991, 1996), probably sensing the direction of the $\mathrm{G}$ vector independently of its magnitude. Thus, if the abdominal graviceptors sense the body orientation in the gravitational field and the neck proprioceptors sense the position of the head with respect to the trunk, this would imply information on the head's position, relative to the direction of gravity. It might be of interest, in this context, to consider the strange sensation reported by one of the test subjects. At $2 \mathrm{G}$, she had the feeling as if the head was tilted backward while the trunk was still upright. If the abdominal graviceptors give a correct message about the position of the trunk with respect to gravity while the otolith organs signal a backward head tilt, these two different sources of graviceptive information would be at conflict with the neck proprioceptors, assuming that the latter are providing correct information about the head's position with respect to the trunk. For the test subject at issue, both of the two different sources of graviceptive information seem to have predominated over neck proprioception.

\section{The Influence of Semicircular Canal Activity on the VPEL}

The semicircular canal stimulus during acceleration of a gondola centrifuge has been described in detail in earlier studies (Glasauer, 1993; Guedry \& Oman, 1990; Guedry, Rupert, McGrath, \& Oman, 1992; McGrath, Guedry, Oman, \& Rupert, 1995). Briefly, during acceleration of the gondola centrifuge the pitch-plane angular velocity stimulus succeeds and overlaps a yaw-plane angular velocity stimulus, and further, it is accompanied by a roll change-in-position canal stimulus corresponding to the inclination of the gondola. Similar vestibular situations occur in natural life - for instance, when a runner is entering a curved path and leans inward in order to compensate for the centrifugal force. Thus, the central nervous system 

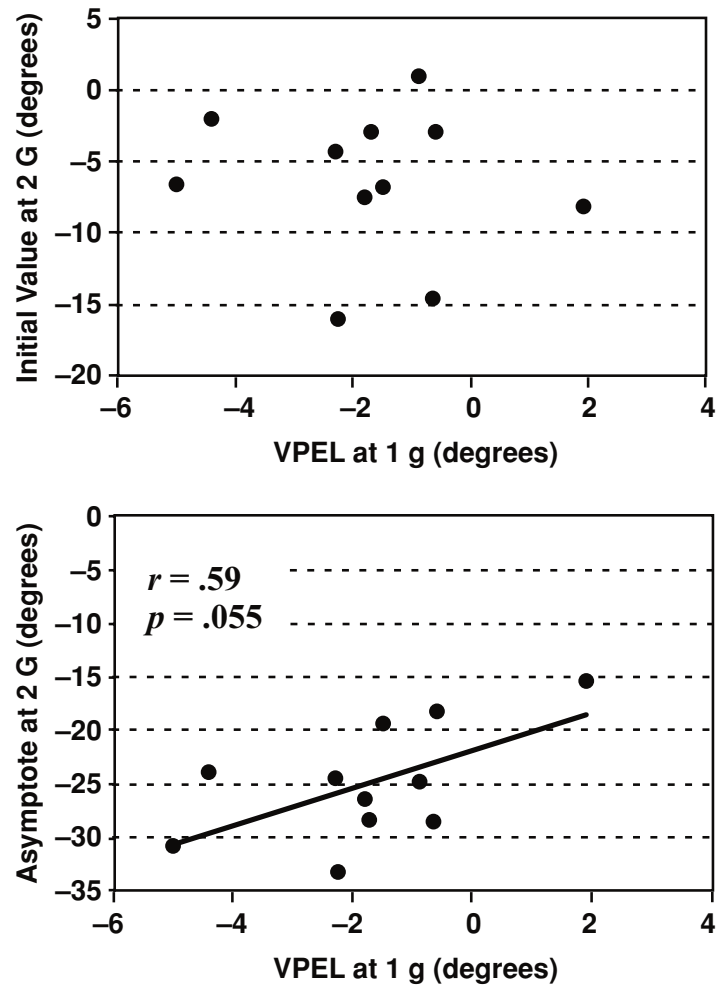

Figure 5. Top panel: The initial value for the visually perceived eye level (VPEL) at $2 \mathrm{G}$ plotted against the value at $1 \mathrm{~g}$. These two variables seem to be independent. Lower panel: The asymptotic value for VPEL at $2 \mathrm{G}$ plotted against the value at $1 \mathrm{~g}$. There tends to be a correlation, so that subjects for whom the VPEL at $1 \mathrm{~g}$ is relatively low will have a larger depression after a period in the 2-G environment. Each point represents one individual.

of a test person might have had possibilities of learning to interpret a stimulus situation of the kind generated in the centrifuge. The stimulus pattern to the semicircular canals would, in an ideal subject, be interpreted as due to a lateral tilt (while in a curve) with respect to the fixed coordinate system provided by the surface of the earth and the direction of gravity.

At the end of acceleration, the major semicircular canal stimulus is pitch-backward angular velocity. It might have been expected that this should enhance a depression of the VPEL by contributing to the utriculus-mediated illusion of backward tilt. However, the depression of the VPEL was obviously inhibited by the semicircular canal stimulation. Two important conclusions may be drawn from this observation. First, when the pitch-backward angular velocity canal stimulus is only one component of the complex canal stimulus occurring during acceleration of the centrifuge, it is not interpreted as due to a backward change in pitch position, suggesting the importance of a complex whole of semicircular canal information for the interpretation of single stimulus components. Second, when an otolithic signal for change in head position with respect to gravity is not accompanied by a corresponding change-in-position signal from the semicircular canals, the otolithic input will not immediately cause a sensation of change in head orientation with respect to gravity.

The latter is of interest as regards the problem of the ambiguity of otolithic information. Due to the equivalence between gravity and inertia, the same otolithic stimulus may be given either by means of static head tilt in one direction or by linear acceleration in the opposite direction. It has been pointed out by Glasauer (1993) that the discrimination between tilt and translation can probably be performed thanks to complementary information from the semicircular canals; a change in head orientation with respect to gravity results in semicircular canal stimulation, whereas a linear acceleration does not. Angelaki, McHenry, Dickman, Newlands, and Hess (1999) have demonstrated, for the rhesus monkey, that when the semicircular canals are inactivated, the vestibulo-ocular system no longer discriminates between otolith stimulation from tilt and from translation.

Earlier studies in gondola centrifuges (Glasauer, 1993; Tribukait, 1999) have demonstrated that when there is a persistent otolithic signal for upright position in the roll plane, a semicircular canal signal for a change in roll position causes only a minor sensation of lateral tilt. The present study is complementary in showing that when an otolithic signal for a change in pitch position is not accompanied by any corresponding semicircular canal information, the perception of pitch tilt is delayed. Altogether, these findings emphasize the importance of a close cooperation between the otolith organs and the semicircular canals in the perception of changes in head orientation with respect to gravity.

The initial deviation of the VPEL may be considered as the outcome of a conflict between, on the one hand, otolithic information on backward head tilt and, on the other hand, semicircular canal information saying that the head is still upright in pitch. Thus, it seems that the brain may correctly interpret the complex semicircular canal input during acceleration of the centrifuge but that this interpretation is forgotten, so that the otolithic illusion of backward head tilt may gradually develop.

In contrast to the initial deviation, the asymptote of the VPEL at $2 \mathrm{G}$, as well as the VPEL in a 1-g environment, is likely to be a rather pure measure of graviceptive function. In addition, these two variables tended to correlate, which also suggests that they are dependent on the same sensory systems.

There were considerable differences between the individuals, especially as regards the initial value for VPEL at $2 \mathrm{G}$ and as regards the time constant. It is certainly of interest, with respect to the disorientation problem in aviation, that normal subjects, forming a rather homogeneous group in a 1-g environment, may respond very differently when exposed to unusual and conflicting vestibular stimulation in the absence of visual cues.

\section{Memory Functions Related to the Semicircular Canals}

During constant speed rotation, the activity of semicircular canal nerve afferents decays exponentially with 
a time constant of 2-6 sec (see Goldberg \& Fernandez, 1984, for references), without any difference between the horizontal and the vertical canals. However, the compensatory (slow) phase of rhythmic eye movements (nystagmus), which serves to stabilize the gaze with respect to the surroundings, has been found to decay with a time constant of 15-20 sec for the horizontal canals; for vertical canal stimulation, the time constant is half of that (see Young, 1984, for references). This increase of the time constant is ascribed to a central nervous function (Baloh, 1989; Young, 1984) often called the velocity storage mechanism (B. Cohen, Matsuo, \& Raphan, 1977; Raphan, Matsuo, \& Cohen, 1979; Robinson, 1977), which is a kind of working memory increasing the time during which eye velocity corresponds to head velocity. This velocity storage mechanism may also influence the duration of certain spatial illusions. It has, for instance, been found that test subjects, seated upright and rotated about a head-centered earth-vertical axis, have a tilt of the subjective visual vertical (Wapner, Werner, \& Morant, 1951) or subjective visual horizontal (SVH) (Wade \& Curthoys, 1997). In the study by Wade and Curthoys, the magnitude of this tilt was $2^{\circ}-10^{\circ}$ initially at an angular velocity of $250 \% \mathrm{sec}$, and it ceased completely within $30-40 \mathrm{sec}$ of constant velocity. This suggests a time constant, $T$, of $10-15 \mathrm{sec}$, which is compatible with the time constant for the velocity storage mechanism. (After $3 \mathrm{~T}$, an exponentially decaying phenomenon approximates the baseline.)

In addition to dynamic responses during angular movements, the semicircular canal system is involved in sensing angular changes in position (Guedry, 1974; Israël, Bron- stein, Kanayama, Faldon, \& Gresty, 1996; Israël, Fetter, \& Koenig, 1993; Metcalfe \& Gresty, 1992). This function is dependent on central nervous integration of the semicircular canal velocity signal over time (Baloh, 1989), a velocity-to-position integrator (Glasauer \& Mittelstaedt, 1998; Mergner, Rumberger, \& Becker, 1996). The usefulness of such information on changes in head position must be dependent on memory functions, especially when there is some latency between a perceived change in angular position and the execution of a spatial orientation task, as well as during such orientation tasks that, in themselves, have a considerable duration.

In an earlier investigation, this memory for changes in position has been studied via measurement of the $\mathrm{SVH}$ during gondola centrifugation (Tribukait, 2003). Initially, after acceleration, there was a tilt of the SVH, the direction of which was compensatory with respect to the inclination of the gondola. However, during constant speed of the centrifuge, this SVH tilt gradually decayed with a time constant of 1-2 min. This slow decay has been interpreted as due to a memory for angular changes in position detected by the semicircular canals - a position storage mechanism (see Figure 6).

The deviation of the VPEL approached its asymptotic value with a time constant that was considerably larger than the time constants for various phenomena related to angular velocity stimulation. Rather, it seems comparable with the time course for the decay of the position storage mechanism. Because of this similarity with respect to time course of the SVH and the VPEL during gondola centrifugation, it seems likely that both of them are re-

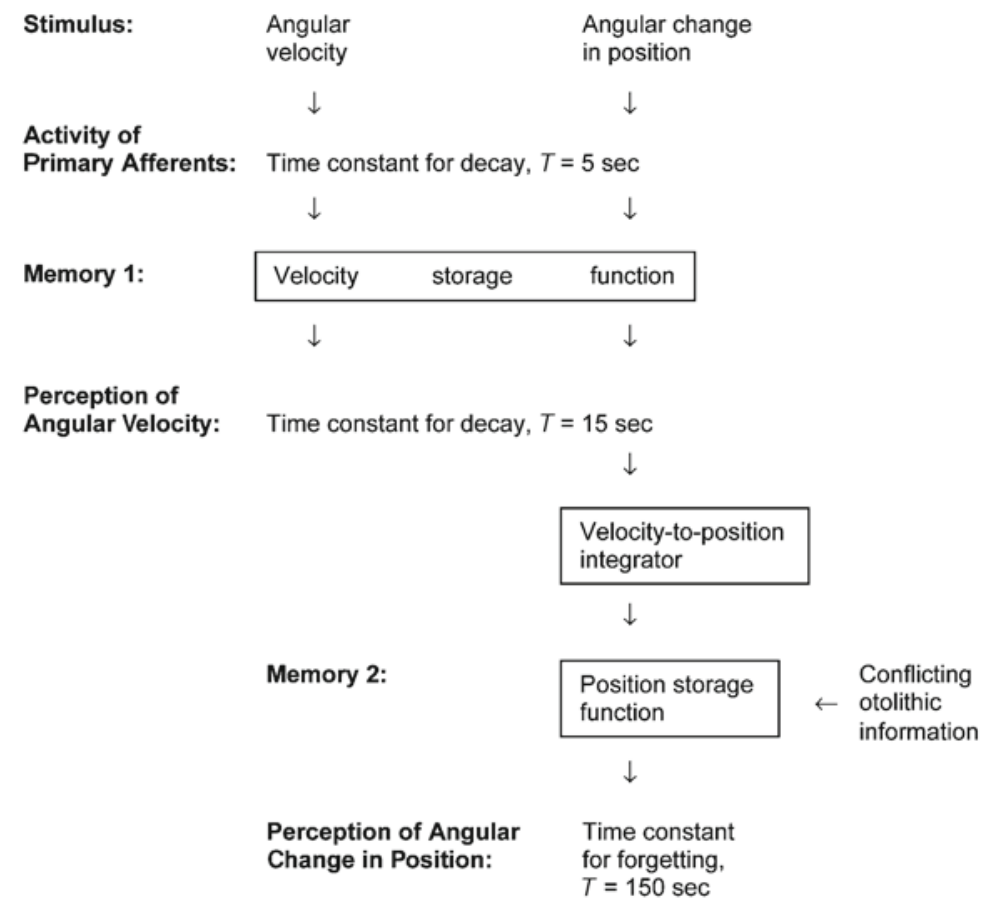

Figure 6. Block diagram showing, in a very simplistic way, the relationships between vestibular stimuli, memory functions, and perceptions. 
lated to a position storage mechanism. However, there is an important difference between the decay of the SVH tilt and the asymptotic increase in the deviation of the VPEL: The SVH tilt reflects an intravestibular conflict in which an initial semicircular canal signal on roll head tilt is counteracted by a persistent otolithic message on upright position. In contrast, for the pitch-plane, the otolithic message on backward head tilt is in conflict with the semicircular canals, which deny that a backward head tilt has occurred.

It should be noted, in this context, that deviations of the VPEL from a baseline (in darkness at $1 \mathrm{~g}$ ) may also be induced by means of exposure to a pitched visual environment. Following exposure, the deviation of the VPEL from the baseline has been found to decay with a time constant of about 4 min (Matin \& Li, 1995). This is compatible with the time constants found in the present study. In both cases, the decay function may be considered as representing a gradual forgetting of a stimulus related to spatial orientation in the pitch plane. It is, therefore, tempting to speculate about common memory functions for visual and vestibular information on changes in orientation.

\section{REFERENCES}

Angelaki, D. E., McHenry, M. Q., Dickman, J. D., Newlands, S. D., \& Hess, B. J. M. (1999). Computation of inertial motion: Neural strategies to resolve ambiguous otolith information. Journal of Neuroscience, 19, 316-327.

BALOH, R. W. (1989). Vestibulo-ocular, cervico-ocular, and visuo-ocular reflexes. Bulletin de la Société Belge d'Ophtalmologie, 237, 35-66.

BECKH, H. VON (1956). Gravity changes in aircraft and ships. Journal of the British Interplanetary Society, 15, 73-81.

Clark, B., Graybiel, A., \& MacCorquodale, K. (1948). The illusory perception of movement caused by angular acceleration and by centrifugal force during flight: II. Visually perceived motion and displacement of a fixed target during turns. Journal of Experimental Psychology, 38, 298-309.

Cohen, B., Matsuo, V., \& Raphan, T. (1977). Quantitative analysis of the velocity characteristics of optokinetic nystagmus and optokinetic after-nystagmus. Journal of Physiology, 270, 321-344.

Cohen, M. M. (1973). Elevator illusion: Influences of otolith organ activity and neck proprioception. Perception \& Psychophysics, 14, 401-406

Correia, M. J., Hixson, W. C., \& Niven, J. I. (1968). On predictive equations for subjective judgments of vertical and horizon in a force field. Acta Oto-Laryngologica, Suppl. 230.

Corvera, J., Hallpike, C. S., \& Schuster, E. H. J. (1958). A new method for the reconstruction of the human macular planes. Acta Oto-Laryngologica, 49, 4-16.

DiZıo, P., Li, W., LaCKNER, J. R., \& Matin, L. (1997). Combined influences of gravitoinertial force level and visual field pitch on visually perceived eye level. Journal of Vestibular Research, 7, 381-392.

Fernandez, C., Goldberg, J. M., \& Abend, W. K. (1972). Response to static tilts of peripheral neurons innervating otolith organs of the squirrel monkey. Journal of Neurophysiology, 35, 978-987.

Glasauer, S. (1993). Human spatial orientation during centrifuge experiments: Non-linear interaction of semicircular canals and otoliths. In H. Krejcova \& J. Jerabek (Eds.), Proceedings of the XVIIth Bárány Society Meeting, Prague 1992 (pp. 48-52). Uppsala, Sweden: Bárány Society.

Glasauer, S., \& Mittelstaedt, H. (1998). Perception of spatial orientation in microgravity. Brain Research Reviews, 28, 185-193.

Goldberg, J. M., \& Fernandez, C. (1984). The vestibular system. In J. M. Brookhart \& V. B. Mountcastle (Section Eds.) \& I. Darian-Smith
(Vol. Ed.), Handbook of physiology: Section 1. The nervous system: Vol. 3. Sensory processes. Bethesda, MD: American Physiological Society.

GuedRY, F. E. (1974). Psychophysics of vestibular sensation. In H. H. Kornhuber (Ed.), Handbook of sensory physiology (Vol. 6, Pt. 2, pp. 1154). Berlin: Springer.

GuEdRY, F. E., \& OMAN, C. M. (1990). Vestibular stimulation during a simple centrifuge run (NAMRL-1353). Pensacola, FL: Naval Aerospace Medical Research Laboratory.

Guedry, F. E., Rupert, A. H., McGrath, B. J., \& Oman, C. M. (1992). The dynamics of spatial orientation during complex and changing linear and angular acceleration. Journal of Vestibular Research, 2, 259-283.

Hoppeler, P. (1913). Über den Stellungsfaktor der Sehrichtungen: Eine experimentelle Studie. Zeitschrift für Psychologie, 66, 249-262.

IsraËL, I., Bronstein, A. M., Kanayama, R., FAldon, M., \& Gresty, M. A. (1996). Visual and vestibular factors influencing vestibular “navigation." Experimental Brain Research, 112, 411-419.

ISRAËL, I., FetTer, M., \& KoENIG, E. (1993). Vestibular perception of passive whole-body rotation about horizontal and vertical axes in humans: Goal-directed vestibulo-ocular reflex and vestibular memorycontingent saccades. Experimental Brain Research, 96, 335-346.

Lindeman, H. H. (1969). Studies on the morphology of the sensory regions of the vestibular apparatus. Berlin: Springer.

Loe, P. R., Tomko, D. L., \& Werner, G. (1973). The neural signal of angular head position in primary afferent vestibular nerve axons. Journal of Physiology, 230, 29-50.

Matin, L., \& Fox, C. R. (1989). Visually perceived eye level and perceived elevation of objects: Linearly additive influences from visual field pitch and from gravity. Vision Research, 29, 315-324.

Matin, L., \& LI, W. (1995). Light and dark adaptation of visually perceived eye level controlled by visual pitch. Perception \& Psychophysics, 57, 84-104.

McGrath, B. J., Guedry, F. E., Oman, C. M., \& Rupert, A. H. (1995). Vestibulo-ocular response of human subjects seated in a pivoting support system during $3 \mathrm{Gz}$ centrifuge stimulation. Journal of Vestibular Research, 5, 331-347.

Mergner, T., Rumberger, A., \& Becker, W. (1996). Is perceived angular displacement the time integral of perceived angular velocity? Brain Research Bulletin, 40, 467-471.

Metcalfe, T., \& Gresty, M. (1992). Self-controlled reorienting movements in response to rotational displacements in normal subjects and patients with labyrinthine disease. In B. Cohen, D. Tomko, \& F. Guedry (Eds.), Sensing and controlling motion: Vestibular and sensorimotor function (Annals of the New York Academy of Sciences, Vol. 656, pp. 695-698). New York: New York Academy of Sciences.

Mittelstaedt, H. (1983). A new solution to the problem of the subjective vertical. Naturwissenschaften, 70, 272-281.

Mittelstaedt, H. (1988). The information processing structure of the subjective vertical: A cybernetic bridge between its psychophysics and its neurobiology. In H. Marko, G. Hauske, \& A. Struppler (Eds.), Processing structures for perception and action (pp. 217-263). Weinheim: Verlag Chemie.

MittelstaedT, H. (1989). The role of the pitched-up orientation of the otoliths in two recent models of the subjective vertical. Biological Cybernetics, 61, 405-416.

Mittelstaedt, H. (1991). The role of the otoliths in the perception of the orientation of self and world to the vertical. Zoologisches Jahrbücher: Abteilung für allgemeine Zoologie und Physiologie, 95, 419425.

Mittelstaedt, H. (1995). New diagnostic tests for the function of utricles, saccules and somatic graviceptors. Acta Oto-Laryngologica, Suppl. 520, 188-193.

Mittelstaedt, H. (1996). The formation of the visual and the postural vertical. In T. Mergner, \& F. Hlavacka (Eds.), Multisensory control of posture (pp. 147-155). New York: Plenum.

Naganuma, H., Tokumasu, K., Окамото, M., Нashimoto, S., \& YAMASHINA, S. (2001). Three-dimensional analysis of morphological aspects of the human saccular macula. Annals of Otology, Rhinology, \& Laryngology, 110, 1017-1024. 
Raphan, T., Matsuo, V., \& Cohen, B. (1979). Velocity storage in the vestibulo-ocular reflex arc (VOR). Experimental Brain Research, 35, 229-248.

RoBINSON, D. A. (1977). Vestibular and optokinetic symbiosis: An example of explaining by modelling. In R. Baker \& A. Berthoz (Eds.), Control of gaze by brainstem neurons (pp. 49-58). Amsterdam: Elsevier.

SchöNe, H. (1964). On the role of gravity in human spatial orientation. Aerospace Medicine, 35, 764-772.

StockWell, C. W., \& GUEDRY, F. E. (1970). The effects of semicircular canal stimulation during tilting on the subsequent perception of the visual vertical. Acta Oto-Laryngologica, 70, 170-175.

TRIBUKAIT, A. (1999). Semicircular canal and saccular influence on the subjective visual horizontal during gondola centrifiguation. Journal of Vestibular Research, 9, 347-357.

Tribukait, A. (2003). Human vestibular memory studied via measure- ment of the subjective horizontal during gondola centrifugation. Neurobiology of Learning \& Memory, 80, 1-10.

WAdE, S. W., \& CurThoys, I. S. (1997). The effect of ocular torsional position on perception of the roll-tilt of visual stimuli. Vision Research, 37, 1071-1078.

Wapner, S., Werner, H., \& Morant, R. B. (1951). Experiments on sensory-tonic field theory of perception: III. Effects of body rotation on the visual perception of verticality. Journal of Experimental Psychology, 42, 351-357.

Young, L. R. (1984). Perception of the body in space: Mechanisms. In S. R. Geiger (Ed.), Handbook of physiology (Sect. 1, Vol. 3, pp. 10231066). Bethesda, MD: American Physiological Society.

(Manuscript received April 21, 2004; revision accepted for publication January 24, 2005.) 